\title{
When does mechanical plantar stimulation promote sensory re-weighing: standing on a firm or compliant surface?
}

\author{
Andrea Preszner-Domjan • Edit Nagy • \\ Edit Szíver · Anna Feher-Kiss · Gyöngyi Horvath • \\ Janos Kranicz
}

Received: 27 June 2011 / Accepted: 3 December 2011/Published online: 15 December 2011

(C) Springer-Verlag 2011

\begin{abstract}
The purpose of this study was to investigate the effect of different types of mechanical stimulation of the sole on standing postural stability in healthy, young adults. Fifty subjects (34 women, 16 men; mean age $23 \pm 2$ (mean \pm SE)) stood barefoot on fixed force plates both with open and closed eyes on firm surface and then on compliant surface (foam). A modified Clinical Test of Sensory Interaction on Balance protocol was employed to assess the center of gravity (COG) excursions along anteroposterior (AP) and mediolateral (ML) axes on each surface and visual condition. After the baseline measurement, a stimulation was applied with an elastic spiked layer topped to the firm and then foam surface, and the COG excursions were measured during the stimulation, and then at least $30 \mathrm{~min}$ after the stimulation of the spiked layer, we used $10 \mathrm{~min}$ of manual static and glide pressure applied to the plantar surface of both feet. Immediately after manual stimulation, static balance parameters were measured again. Results showed that after manual stimulation, the sway path with closed eyes decreased significantly on the AP and ML directions on firm surface conditions.
\end{abstract}

Communicated by Dick F. Stegeman.

A. Preszner-Domjan $(\bowtie) \cdot$ E. Nagy · E. Szíver · A. Feher-Kiss Department of Physiotherapy, Faculty of Health Sciences and Social Studies, University of Szeged, Temesvári krt. 31, Szeged 6726, Hungary

e-mail: andrea@etszk.u-szeged.hu

G. Horvath

Department of Physiology, Faculty of Medicine, University of Szeged, Szeged, Hungary

J. Kranicz

Department of Orthopaedics, Medical School, University of Pécs, Pécs, Hungary
The spiked layer caused significantly decreased sway path on firm platform in both directions, but it was ineffective on compliant surface. Our results established that the activation of plantar mechanoreceptors by $10-\mathrm{min}$ manual stimulation can partially compensate subjects for the absence of visual input and the lack of accurate pressure information from the supporting surface, too.

Keywords Postural sway - Sole - Manual stimulation · Spiked layer $\cdot$ Compliant surface

\section{Introduction}

Control of balance is a very important condition for normal movement and activities of daily life. Balance impairments are the leading causes of falls, which are very common and severe problems of the elderly leading to life threatening injuries. Postural orientation and stability as postural control is the result of a controlling process of the body position in space that includes various sensory motor and cognitive components.

Despite the availability of multiple sensory inputs (visual, vestibular and somatosensory), the central nervous system (CNS) generally relies mainly on one sense at a time for orientation (Nashner 1982). Thus either the visual information (Lee and Lishman 1977), or the proprioception and the cutaneous inputs are important for maintaining the body's equilibrium in a static state (Nashner et al. 1982).

Studies of multisensory integration and postural control suggested a reweighing process in response to changes in the availability of sensory conditions (Nashner et al. 1982; Woollacott et al. 1986; Oie et al. 2002). These results support the concept of hierarchical weighing of sensory information for postural control based on their accuracy in 
reporting the position or movements of the body, i.e., when a sense is not providing accurate information regarding the body position, the CNS modifies the relative importance of the sense for postural control.

Changes in a quiet stance position are often related to a change in pressure under the feet. The slow adapting mechanoreceptors of the sole are able to code the static pressures applied on their receptive fields (Vedel and Roll 1982). As Kavounoudias et al. (1998) have described, the plantar sole is a 'dynamometric map' for balance control. Due to their anatomical location that interfaces between the sole and the ground, the plantar cutaneous mechanoreceptors provide detailed information about the supporting surface properties and position and movement of body related to supporting surfaces. Accordingly, pressure changes from the main supporting areas of the feet present continuous and accurate information about the displacement of the body.

Over the past few decades, researchers have focused on the importance of tactile and pressure information from the plantar surface of the feet. Considerable researches have supported the role of the somatosensory and proprioceptive inputs from the legs, applying provoked reduction of afferent information from the lower limb with vascular ischemia, anesthesia or cooling. Decrease in the somatosensory information leads to a degradation of stability resulting in an increased postural sway during quiet or perturbed stance (Mauritz and Dietz 1980; Hayashi et al. 1988; Magnusson et al. 1990; Horak et al. 1990; Meyer et al. 2004).

In recent years, studies have demonstrated that an additional somatosensory input has positive effects on postural control by using various stimulation methods on the sole such as massage or changing the characteristics of the supporting surface (Nurse et al. 2005; BernardDemanze et al. 2006, 2009; Palluel et al. 2008, 2009; Vaillant et al. 2008, 2009).

These results confirm that instrumental or manual stimulation of the plantar sole and the special surfaces have an impact on the mechanoreceptors of the feet, and this relevant tactile information helps to realize the body position on firm surface when vision is not available. Nevertheless, the question arises regarding the effect of mechanical stimulation on postural control in patients with reduced visual acuity. Goldreich and Kanics (2003) have compared passive tactile acuity of blind and sighted subjects, and confirmed that blind individuals show superior abilities in the use of tactile information. Jeka and his colleagues (1996) have supported that tactile information from contact with a cane reduced postural sway in blind individuals. However, we could not find data in literature on how mechanical stimulation of the feet influences postural control in patients with reduced visual acuity.
Studies which searched the effect of mechanical stimulation of the feet have primarily focused on the effect of various mechanical stimulations on stable, firm surface. Nevertheless, standing on an unstable, compliant surface, for example on foam, impairs the accuracy of the information coming from the cutaneous mechanoreceptors of the soles. Wu and Chiang (1996) have investigated alterations in the pressure distribution and contact area under the feet related to changes in compliance of the supporting surface. They found close correlation between the plantar pressure and the surface compliance suggesting that sensory input to the mechanoreceptors would be decreased by the added layers of foam.

The question arises if sole manual stimulation or an added stimulating surface provides enough pressure input to induce re-weighing of sensory information on the foam surface when visual information is absent. We hypothesized that inaccurate and reduced pressure information caused by a compliant foam surface would be compensated by activation of cutaneous mechanoreceptors of the sole with different mechanical stimulations.

Therefore, the first aim of our study was to assess the positive effect of manual stimulation on the sole concerning postural stability on firm and also on foam surfaces in healthy young adults. We used only manual stimulation without joint mobilization to increase the sensory inputs from the mechanoreceptors of the sole. The goal of the stimulation was to activate mainly the slowly adapting cutaneous mechanoreceptors (Merkel's discs and Ruffinilike endings), which detect touch, pressure and stretching of the skin, and which are able to code static and dynamic changes in pressure applied to their receptive fields (Vedel and Roll 1982). We also hypothesized that manual mechanical stimulation would generate smaller postural sway not only on firm surface but also on foam surface, especially when visual input is absent.

An additional goal of this study was to explore the prompt effect of standing on rough surface by adding a special spiked layer to the force platform. We investigated whether a direct stimulating effect of an added spiked layer would influence the static balance parameters on a firm and compliant (foam) supporting surface similarly to manual stimulation.

\section{Methods}

Subjects

Fifty young, healthy adults [34 women and 16 men; mean age $23 \pm 2$; mean body weight $67 \pm 9.5 \mathrm{~kg}$, mean height $170 \pm 7.1 \mathrm{~cm}$, mean BMI $22.81 \pm 2.401 \mathrm{~kg} / \mathrm{m}^{2}$ (mean \pm $\mathrm{SE})$ ] volunteered for this study. None were excluded for 
having any acute illness, diagnosed neurological or musculoskeletal disease, any known balance impairment or current use of medication that could have affected their balance control. All subjects gave their informed consent prior to participation in the study, which was approved by the local institutional Ethics Committee.

To exclude the loss of plantar sensitivity, we used the SenseLab Aesthesiometer (Semmes and Weinstein monofilaments) at six plantar regions (heel, lateral side of midfoot, first, third, fifth metatarsal heads and hallux) to evaluate the perception threshold of the mechanoreceptors of the sole to light touch. We applied a modified 4-2-1 step algorhythm that has been described by Dyck et al. (1993). Assessment of plantar sensitivity was performed a day before the stimulating interventions and the posturographic measurements. $0.21 \mathrm{~g}$ nominal force was adapted as normal touch threshold for the sole of the foot (Bell-Krotoski et al. 1995).

\section{Static balance measurement}

The clinical test of sensory interaction on balance (CTSIB) designed by Shumway-Cook and Horak (1986) allows manipulation of the senses and investigation of the effect of visual, vestibular and somatosensory inputs on standing balance. The CTSIB uses different conditions to test how people adapt their senses to changing sensory conditions during the maintenance of stance.

We used the modified CTSIB by NeuroCom to perform the test on a computerized force plate to measure the body sway via horizontal excursions of the COG. There are four tasks in CTSIB: eyes open and firm surface, eyes closed and firm surface, eyes open and foam surface, and eyes closed and foam surface.

Static postural stability was measured during quiet standing on a fixed dual force plate (NeuroCom Basic Balance Master). To offer control data, posturographic measures were performed for each task. The COG excursions along anteroposterior (AP) and mediolateral (ML) axes were measured under various visual and surface conditions. First, subjects stood barefoot on firm surface with eyes open (EO) and then eyes closed (EC). The measurement was performed in three sessions $(3 \times 10 \mathrm{~s})$ in both visual conditions. To reduce tactile and pressure information from the mechanoreceptors of the sole, we altered the support surface from the firm, level force plate to a compliant foam pad, and the measurement was repeated in both eye conditions in three sessions. We used the NeuroCom square foam balance assessment pad (size $46 \times 46 \times 13 \mathrm{~cm})$.

For all conditions, subjects stood quietly with arms comfortably and loosely by their sides, and were asked to look forward in the EO condition and to close their eyes in the EC condition. Their feet were positioned by the signs of the NeuroCom platform, the distance between the midline of the heels changed from 22 to $30 \mathrm{~cm}$ according to the subjects' height. Toe-out was positioned to subjects' comfort.

\section{Interventions}

\section{Spiked layer}

To investigate the prompt effect of a stimulating surface on postural stability, we used a special, spiked layer during posturographic measurement. It was a thin elastic layer of rubber with spiked layer (density 5 spikes $/ \mathrm{cm}^{2}$, height of spikes $7 \mathrm{~mm}$, diameter of spikes $2 \mathrm{~mm}$ ), and it was placed onto the force plate. Subjects were asked to stand still bare feet with arms comfortably at their sides, and their feet were adjusted according to the signs of the platform. Subjects did not report any discomfort because of the spiked layer. The displacement of COG was measured while subjects stood on the stable platform covered with the special layer. The measurements were performed in three sessions $(3 \times 10 \mathrm{~s})$ in both open and closed eye conditions. Then we altered the supporting surface from the firm to foam one with the added spiked layer, first with open eyes and then with closed eyes. Thus, the spiked layer affected the sole just during the posturographic measurement. The baseline measurement of static postural stability without any stimulation served as a control condition.

\section{Manual stimulation}

Manual stimulation was performed by two assistants (25-25 subjects). To standardize the intensity of stimulation, we used a learning process. Pressure stimulation was applied on both feet at the same time by the two assistants, and the subject checked if the pressure was equal on both soles. It was repeated three times with three different subjects, and then the force of the manual pressure was measured by a strength dynamometer (Chatillon MSE 100). The mean force of pressure was $48 \mathrm{~N}$, which was determined as a standard force for glide and static pressure during the stimulation. The learning procedure guaranteed that the manual stimulation force produced by the two assistants was the same, and the force by their right and left hands was also equal.

Manual technique included static and glide pressure focus on the supporting surfaces of the sole, especially over the heel region, and the region of metatarsal heads. It was applied for $10 \mathrm{~min}$, while subjects were relaxing in a sitting position and their feet were supported. Stimulation was performed simultaneously on both feet by an assistant. 
Static balance parameters were measured before and after manual stimulation. The baseline measurement of the static postural stability without any stimulation served as a control condition. Participants were asked to stand up for $20 \mathrm{~s}$ after the stimulation, in this way the negative effect of sudden standing up was avoided. Manual stimulation was applied at least $30 \mathrm{~min}$ after the stimulation of the spiked layer.

\section{Reliability of the measurement}

We compared the results of the three sessions of measurement and found that the sway path of the first session was significantly longer than the sway path of the second or the third session in all measurement conditions; however, there were no significant differences between the results of the second and the third sessions. To ensure the reliability of the measurement, we excluded all data of the first sessions, and the mean of the second and the third assessments were analyzed.

\section{Statistical analysis}

\section{Tactile threshold}

Because of the discrete nature of the data of monofilament diameter, plantar tactile threshold was determined by the median of the nominal force that was felt in each plantar region.

The Wilcoxon Signed Ranks test was used to compare tactile threshold medians of left and right feet with normal touch threshold of the sole.

\section{Sway path}

The following formulas (1 and 2) were applied to calculate the sway paths in ML $(x)$ and $\operatorname{AP}(y)$ directions, where $n$ is the total number of samples, $i$ is the numbering, $s_{y}$ is the path length of A/P sways and $s_{x}$ is the path length of the lateral displacements of $\mathrm{COG}$ :

$$
\begin{aligned}
& s_{x}=\sum_{i=1}^{n-1} \sqrt{\left(x_{i+1}-x_{i}\right)^{2}} \\
& s_{y}=\sum_{i=1}^{n-1} \sqrt{\left(y_{i+1}-y_{i}\right)^{2}}
\end{aligned}
$$

Data were subjected to variance analysis with Statistica 8. to make comparisons between the types of stimulation, and the experimental conditions considered as independent variables. We used two-way ANOVA to analyze sway data on firm and foam surfaces to evaluate the main effects and the interactions of the two visions (eyes open and eyes closed) and three stimulation (baseline, manual stimulation, spiked layer) factors. The post hoc test was the NewmannKeuls test. A level of significance of $p<0.05$ was adapted.

\section{Results}

Tactile sensitivity

Comparing the medians of the tactile threshold in every investigated sole region on both feet with the normal touch threshold of the sole, no significant difference (left sole $p=0.345$, right sole $p=0.916$ ) and loss of sensitivity was observed.

\section{Static balance parameters}

\section{Results of baseline measurement}

The sway paths increased significantly in both directions and in both surface conditions when there was no visual input (Figs. 1, 2). Standing on foam surface significantly increased the sway path in both directions, in both eye conditions compared with standing on firm surface.

The effect of the 10-min manual stimulation and the spiked layer on the balance parameters on firm surface

A main effect of vision was observed in both directions (AP $p<0.001$; ML $p<0.001)$ in the baseline measurement: the absence of visual input (EC) caused a significant increase in the sway path, however, these changes disappeared in the AP and ML directions after manual stimulation, and on the spiked layer in ML direction (Figs. 1a, b).

Results showed a significant two-way interaction of vision $x$ stimulation for the sway path in both AP $(p<0.001)$ and ML $(p<0.001)$ directions on firm surface.

The main effect of manual stimulation was observed in both AP $(p<0.001)$ (Fig. 1a) and ML $(p<0.001)$ (Fig. 1b) directions when subjects stood on firm platform with closed eyes compared to baseline data, i.e., the sway paths decreased significantly. We noticed an additional main effect of stimulation, the spiked layer caused significant decreased sway path in both AP $(p<0.001)$ (Fig. 1a) and ML $(p<0.001)$ (Fig. 1b) directions in EC condition. In ML direction, this effect was more prominent, thus, subjects were fully compensated by the spiked layer for the missing visual information.

In EO condition, the manual stimulation and the spiked layer did not cause any alteration in either the AP or the ML direction. 

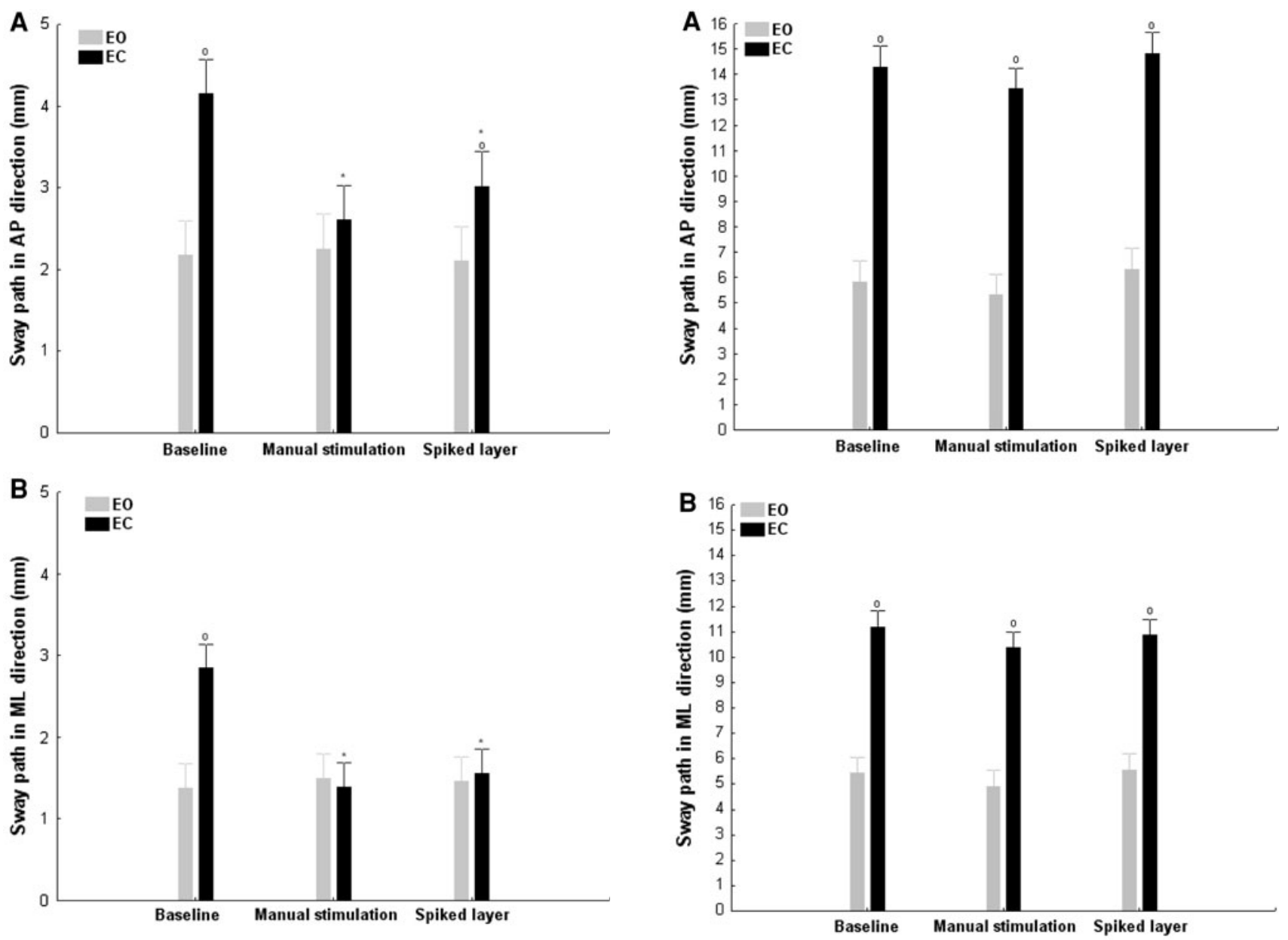

Fig. 1 The effect of the 10-min manual stimulation and the spiked layer on postural stability when standing on firm surface. The sway path (mean \pm SE) in AP (a) and ML directions (b) with open and closed eyes ( $E O$ eyes open, $E C$ eyes closed). Statistically significant differences $(p<0.05)$ in comparison with the open eye condition (circle); and in comparison with the baseline measurement (asterisk)

The effect of the 10-min manual stimulation and the spiked layer on the balance parameters on foam surface

On the foam surface, a main effect of vision was observed in all stimulation conditions in both directions: standing on foam surface with closed eyes caused significant increase in the sway paths in both directions in the baseline measurement, after manual stimulation and in the spiked layer measurement as well.

In contrast to the firm surface, the analysis revealed no interaction of vision $x$ stimulation for the sway path in both directions on foam surface. However, a main effect of manual stimulation was observed, the sway path decreased in ML and AP directions in EC condition (Fig. 2a, b) in comparison with the sway path before the stimulation.

Compared with the baseline data, the spiked surface did not change the sway paths substantially; it remained

Fig. 2 The effect of the 10-min manual stimulation and the spiked layer on postural stability when standing on foam surface. The sway path (mean \pm SE) in AP (a) and ML directions (b) with open and closed eyes ( $E O$ eyes open, $E C$ eyes closed). Statistically significant differences $(p<0.05)$ in comparison with the open eye condition (circle); and in comparison with the baseline measurement (asterisk)

roughly unchanged in both directions and in both eye conditions (Fig. 2a, b).

\section{Discussion}

In the present study, our subjects' ability to remain stable in quiet stance was evaluated under various conditions. Thus, we altered the availability of visual information and pressure and tactile information coming from the supporting surface. Several studies have investigated the organization of sensory input for postural control. Considerable researches have shown that visual input plays a significant role in balance control (Lee and Lishman 1977; Brandt et al. 1986). However, when visual information is unavailable but the somatosensory and vestibular information is available and accurate, then the individual will 
have to rely primarily on the somatosensory input and only secondarily on the vestibular input.

Horak et al. (1990) have examined the postural stability of neurologically intact adults and patients with loss of vestibular function with the CTSIB. Their results show that instability occurred in patients with vestibular problem when visual input was not available and somatosensory input was disturbed. Accordingly, when the visual information is unavailable and the somatosensory information is inaccurate, the individual will have to rely predominantly on the vestibular input.

Our results demonstrated that in case of baseline measurements, the sway paths increased due to the absence of visual input and also when somatosensory information was reduced by the foam surface. Changes in the sway paths under various sensory conditions are in line with the results of Woollacott et al. (1986). However, the spiked layer and manual stimulation of the sole resulted in improvement in the postural control in EC condition, activation of the plantar mechanoreceptors partially compensated subjects for the lack of vision on the firm surface in both directions, and manual stimulation also caused significantly decreased sway path on the foam surface in both directions in EC condition.

Previous studies confirm both in young and elderly people that different plantar stimulations improved postural control on a firm surface and in absence of vision (Maki et al. 1999; Maurer et al. 2001; Priplata et al. 2003; Nurse et al. 2005; Bernard-Demanze et al. 2006; Vaillant et al. 2008; Palluel et al. 2008).

Vaillant et al. (2008) have measured the postural stability of 17 elderly subjects before and after a 20-min therapeutic manipulation that involved manual massage of the feet and mobilization of both foot and ankle joints. During posturographic measurement, the availability of visual input was altered to detect the immediate effect of the suppression of vision and the postural adaptation over time to the absence of vision. Their results showed that after massage and mobilization of the feet and ankles, postural stability remained unchanged when the vision was absent compared to the baseline values without stimulation. Thus, therapeutic manipulation allows the elderly to be partially compensated for the destabilising effect induced by the suppression of vision. Further investigations have shown that plantar massage and joint mobilization of the feet and ankles produce significant improvement in the results of the clinical tests of balance in elderly subjects (Vaillant et al. 2009).

Our results were in line with Vaillant et al., as manual stimulation of the plantar sole improved postural stability on firm surface; indeed the absence of vision did not influence the sway path in any direction with respect to the eyes open condition. Nonetheless, in contrast to their stimulating method, we used only manual stimulation of the sole to enhance somatosensory information from the feet, thus, our results demonstrated that manual static and glide pressure is effective by itself in facilitating postural stability in quiet stance.

Furthermore, Bernard-Demanze et al. (2006) have investigated the effect of three sessions of 10-min massage by a massage device applied under the feet. Static balance parameters of 19 healthy young adults have been measured on firm platform in EC condition. Their findings have shown that each massage causes a decrease in vertically projected motion of COG along the ML axis on stable surface without visual input. In agreement with this study, we found that the 10-min stimulation of the plantar sole would contribute to a better evaluation of the pressure information from the supporting surface and would facilitate postural stability on firm surface.

An interesting finding of our study was that the $10-\mathrm{min}$ manual stimulation of the sole significantly decreased the sway paths on firm surface, and there is a slight decrease on foam surface also in both AP and ML directions when vision was not available.

In an earlier study, Chiang and Wu (1996) have pointed out that standing on foam would affect the inputs to cutaneous mechanoreceptors coming from the supporting surface. They found close correlation between plantar pressure and surface compliance suggesting that the input to mechanoreceptors would be deteriorated by adding layers of foam. Their findings indicate that pressure on the soles seems to play an important role in postural control in quiet stance on firm surface; however, pressure input from the soles becomes less significant on compliant surface. Thus, tactile and pressure information from the plantar mechanoreceptors are reduced or inaccurate during standing on foam surface. Results of the above mentioned study suggest that the compliant surface produces reduced somatosensory information for the feet, thus, mainly visual and vestibular inputs can assist orientation during standing on foam. When standing on foam in EC condition, the vestibular information is accurate, but the lack of vision and the decreased somatosensory inputs generate an enhanced sway path.

We presumed that mechanical stimulation of the plantar sole would provide an efficient activation of plantar mechanoreceptors and can compensate for the lack of vision on the firm surface, and for the lack of visual input and inaccurate somatosensory information on the foam surface, too. The question arises whether our interventions did stimulate the cutaneous mechanoreceptors as well as the proprioceptive receptors of the plantar muscles. As the mechanical stimulations (manual intervention and spiked layer) were performed in the region of the sole, we could have an effect on the proprioceptive receptors in the 
intrinsic muscles of the sole, thus, we cannot fully exclude that the alterations in the muscle tone might have been an impact on postural reactions. Further research is needed to clarify our knowledge on the reasons of improvement in postural stability.

Our results suggested that the activation of the plantar mechanoreceptors by a 10-min manual stimulation can partially compensate subjects for the lack of vision and the inaccurate pressure information from the supporting surface.

The question arises whether positive changes in postural control due to mechanical stimulation of the feet can be attributed to an increase in the sensitivity of plantar receptors. In our study, we neither studied the changes in the plantar tactile threshold due to mechanical stimulation nor did we find data in literature on how tactile sensitivity is influenced by mechanical stimulation of the sole in healthy young subjects.

We observed an important change in ML direction. Winter et al. (1993, 1996) have suggested that the mediolateral sway during quiet standing is concerned as the result of the loading and unloading of the left and right sides. Accordingly, the COG motions along the ML axis mostly depend on body weight distribution over the feet (Winter 1995). Similarly to the results of Bernard-Demanze et al. (2006), our findings supported the idea that plantar sole manual stimulation caused better control of body weight distribution over the two feet.

Lateral stability or instability as an important marker of balance in older persons has been highlighted by several studies (Maki and McIlroy 1996, 1997). In our earlier studies, we found significantly lower ML power in ironmen trained in extreme strenuous exercise in comparison with healthy, normal control subjects, indicating a higher level of motor control in ML direction (Nagy et al. 2004). The ML balance parameters appeared to be more sensitive to the effect of an 8-week balance training in comparison with AP parameters in elderly subjects (Nagy et al. 2007).

Henry et al. (2001) have suggested that the width of the feet position during the posturographic measurement changes the relationship of the center of body mass relative to the limits of stability of the feet and increases the passive stability of the musculoskeletal system. Interestingly, in our study, the postural sway decreased in the ML direction after manual stimulation in both surface conditions, although the distance between the midline of the heels during the posturographic measurement was at least $22 \mathrm{~cm}$. Thus, the width of the stance was likely to extend the passive stability in the ML direction. Based on our findings, it may be hypothesized that improvement of mediolateral control via activation of the plantar mechanoreceptors could be useful from a therapeutic point of view, and it may be an option for decreasing the risk of falls in the elderly.

A further aim of our study was to investigate the direct effect of an additional spiked layer to the force plate on static balance parameters. Our research data showed that the stimulating effect of this spiked layer could trigger a decrease in the sway path in both directions on the firm surface in closed eye condition.

Nurse et al. (2005) have described that a textured shoe insert can cause changes in muscle activity by modified sensory feedback from the sole.

Palluel et al. (2008) have investigated the effect of wearing sandals equipped with spike insoles on sway parameters in healthy young and elderly subjects with relatively intact plantar cutaneous sensation. In their study, participants have stood on the force plate with and without spike insoles for $5 \mathrm{~min}$ in the standing session, and then subjects walked with and without spike insoles in the walking session, and postural stability was measured in each case. These results support that immediately after subjects insert the spike insoles, they exhibit no adaptation, but standing or walking for 5 min with spiked sandals can lead to significant improvement in balance in both groups for AP and ML directions.

Similarly to the above mentioned study, our findings supported the idea that spiked layer could contribute to the improvement of static balance parameters on firm surface.

However, in contrast with manual stimulation, the spiked layer proved to be ineffective on foam surface, the short-term and less intense impact did not seem to have a compensating effect on the alteration of pressure input from the compliant surface.

In our study, the different mechanical stimulations were effective only in closed eye condition. The absence of the effect in open eye condition may be due to the redundancy of senses, namely the CNS used primarily the accurate visual and vestibular inputs, and the stimulated somatosensory input was used only secondarily. Without vision, increased information from the mechanoreceptors became dominant for achieving stability. The studies of Vaillant et al. (2008), Bernard-Demanze et al. (2006) and Palluel et al. (2008), along with the present one, have supported the idea that activated cutaneous information of the sole can partially compensate subjects for the absence of visual input.

The ability of postural control system to re-weigh the available sensory inputs is a generally accepted idea (Stål et al. 2003; Vuillerme and Pinsault 2007; Vuillerme et al. 2008). We would believe that the decrease of sway paths in closed eye condition after the mechanical stimulation showed the adaptive mechanism of the CNS, as it used the facilitated input from the plantar mechanoreceptors as an alternative sensory input for orientation. 


\section{Conclusions}

The present findings demonstrate that manual stimulation is an effective intervention to enhance balance control especially on firm surfaces in young, healthy adults, only when visual information is not available. Manual stimulation was found to have a beneficial effect on the results on the foam surface. It should still be determined whether the benefit of facilitation is preserved over a longer period of time. Manual stimulation caused improved postural stability in both AP and ML directions, which would be due to the better control of body weight distribution over the two feet. It should, therefore, be sensible to investigate whether stimulation of only one of the two feet leads to changes in the dynamics of weight bearing between the feet in quiet stance.

The effect of different mechanical stimulations is likely to be caused by the increased sensitivity of the mechanoreceptors of the plantar sole. The limitation of the experiment is that the effect of the stimulations on tactile sensitivity of the sole was not assessed. Future research is needed to measure tactile threshold before and after plantar mechanical stimulation.

We identified the immediate effects of the spiked layer, but this positive effect appeared on its own on the firm surface without visual input. The rough, spiked layer can help subjects to orientate their body position vertically and to the base of the support.

Our findings showed that young adults can benefit from mechanical stimulation of the sole. Further research is planned to investigate mechanical stimulation effectivity on compliant surface in the elderly or in case of disease related sensory loss.

Our results suggest that stimulation of the plantar cutaneous mechanoreceptors is an effective method, which can easily be used either as a manual intervention in physiotherapy or it may be used as a shoe insert (stimulating spiked layer), as it may help us in decreasing the risk of falls.

Acknowledgments The authors are grateful to Cs. Keresztes for linguistic correction of the manuscript. The authors would like to thank all participants. We declare that the experiments reported in this paper fully comply with all the current Hungarian laws and regulations.

Conflict of interest The authors declare that they have no conflict of interest.

\section{References}

Bell-Krotoski JA, Fess EE, Figarola JH, Hiltz D (1995) Threshold detection and Semmes-Weinstein monofilaments. J Hand Ther $8: 155-162$

Bernard-Demanze L, Vuillerme N, Berger L, Rougier P (2006) Magnitude and duration of the effects of plantar sole massages on the upright stance control mechanism of healthy individuals. Int SportMed J 7:154-169

Bernard-Demanze L, Vuillerme N, Ferry M, Berger L (2009) Can tactile plantar stimulation improve postural control of persons with superficial plantar sensory deficit? Aging Clin Exp Res 21:62-68

Brandt T, Paulus W, Straube A (1986) Vision and posture. In: Bles W, Brandt $\mathrm{T}$ (eds) Disorders of posture and Gait. Elsevier, Amsterdam, pp 157-175

Dyck PJ, O'Brien PC, Kosanke JL, Gillen DA, Karnes JL (1993) A 4 , 2 , and 1 stepping algorithm for quick and accurate estimation of cutaneous sensation threshold. Neurology 43:1508-1512

Goldreich D, Kanics IM (2003) Tactile acuity is enhanced in blindness. J Neurosci 23:3438-3445

Hayashi R, Miyake A, Watanabe S (1988) The functional role of sensory inputs from the foot: stabilizing human standing posture during voluntary and vibration-induced body sway. Neurosci Res 5:203-213

Henry SM, Fung J, Horak FB (2001) Effect of stance width on multidirectional postural responses. J Neurophysiol 85:559570

Horak FB, Nashner LM, Diener HC (1990) Postural strategies associated with somatosensory and vestibular loss. Exp Brain Res 82:167-177

Jeka JJ, Easton RD, Bentzen BL, Lackner JR (1996) Haptic cues for orientation and postural control in sighted and blind individuals. Percept Psychophys 58:409-423

Kavounoudias A, Roll R, Roll JP (1998) The plantar sole is a 'dynamometric map' for human balance control. Neuroreport 9:3247-3252

Lee DL, Lishman JR (1977) Vision, the most efficient source of proprioceptive information for balance control. Agressologie 18:83-94

Magnusson M, Enbom H, Johansson R, Pyykko I (1990) Significance of pressor input from the human feet in anterior-posterior postural control. The effect of hypothermia on vibration induced body sway. Acta Otolaryngol Stockh 110:182-188

Maki BE, McIlroy WE (1996) Postural control in the older adult. Clin Geriatr Med 12:635-658

Maki BE, McIlroy WE (1997) The role if limb movements in maintaining upright stance: the "change in support" strategy. Phys Ther 77:488-507

Maki BE, Perry SD, Norrie RG, McIlroy WE (1999) Effects of facilitation of sensation from plantar foot-surface boundaries on postural stabilization in young and older adults. J Gerontol A Biol Sci Med Sci 54:M281-M287

Maurer C, Mergner T, Bolha B, Hlavacka F (2001) Human balance control during cutaneous stimulation of the plantar soles. Neurosci Lett 302:45-48

Mauritz KH, Dietz V (1980) Characteristics of postural instability induced by ischemic blocking of leg afferents. Exp Brain Res 38:117-119

Meyer PF, Oddson LI, De Luca CJ (2004) The role of plantar cutaneous sensation in unpertubed stance. Exp Brain Res 156:505-512

Nagy E, Toth K, Janositz G, Kovacs G, Feher-Kiss A, Angyan L, Horvath G (2004) Postural control in athletes participating in an ironman triathlon. Eur J Appl Physiol 92:407-413

Nagy E, Feher-Kiss A, Barnai M, Preszner-Domján A, Angyan L, Horvath G (2007) Postural control in elderly subjects participating in balance training. Eur J Appl Physiol 100:97-104

Nashner LM (1982) Adaptation of human movement to altered environments. Trends Neurosci 5:351-361

Nashner LM, Black FO, Wall C (1982) Adaptation to altered support and visual conditions during stance: patients with vestibular deficits. J Neurosci 5:117-124 
Nurse MA, Hulligar M, Wakeling JM, Nigg BM, Stefanyshyn DJ (2005) Changing the texture of footwear can alter gait patterns. J Electromyogr Kinesiol 15:496-506

Oie KS, Kiemel T, Jeka JJ (2002) Multisensory fusion: simultaneous re-weighing of vision and touch for the control of human posture. Cog Brain Res 14:154-176

Palluel E, Nougier V, Olivier I (2008) Do spike insoles enhance postural stability and plantar-surface cutaneous sensitivity in the elderly? Age 30:53-61

Palluel E, Nougier V, Olivier I (2009) The lasting effects of spike insoles on postural control in the elderly. Behav Neurosci 123:1141-1147

Priplata AA, Niemi JB, Harry JD, Lipsitz LA, Collins JJ (2003) Vibrating insoles and balance control in elderly people. Lancet 362:1123-1124

Shumway-Cook A, Horak FB (1986) Assessing the influence of sensory interaction on balance: suggestion from the field. Phys Ther 66:1548-1550

Stål F, Fransson PA, Magnusson M, Karlberg M (2003) Effects of hypothermic anesthesia of the feet on vibration-induced body sway and adaptation. J Vestib Res 13:39-52

Vaillant J, Vuillerme N, Janvey A, Louis F, Braujou R, Juvin R, Nougier V (2008) Effect of manipulation of the feet and ankles on postural control in elderly adults. Brain Res Bull 75:18-22

Vaillant J, Rouland A, Martigné P et al (2009) Massage and mobilization of the feet and ankles in elderly adults: effect on clinical balance performance. Man Ther 14:661-664
Vedel JP, Roll JP (1982) Response to pressure and vibration of slowly adapting cutaneous mechanoreceptors in the human foot. Neurosci Lett 34:289-294

Vuillerme N, Pinsault N (2007) Re-weighting of somatosensory inputs from the foot and the ankle for controlling posture during quiet standing following trunk extensor muscles fatigue. Exp Brain Res 183:323-327

Vuillerme N, Chenu O, Pinsault N, Fleury A, Demongeot J, Payan Y (2008) Can a plantar pressure-based tongue-placed electrotactile biofeedback improve postural control under altered vestibular and neck proprioceptive conditions? Neuroscience 155:291-296

Winter DA (1995) Human balance and posture control during standing and walking. Gait Posture 3:193-214

Winter D, Prince F, Stergiou P, Powel C (1993) Medial-lateral and anterior-posterior motor responses associated with center of pressure changes in quiet standing. Neurosci Res Comm 12:141-148

Winter DA, Prince F, Frank JS Powell C, Zabjek KF (1996) Unified theory regarding $\mathrm{A} / \mathrm{P}$ and $\mathrm{M} / \mathrm{L}$ balance in quiet stance. J Neurophysiol 75:2334-2343

Woollacott MH, Shumway-Cook A, Nashner L (1986) Aging and posture control: changes in sensory organization and muscular coordination. Int J Aging Hum Dev 23:97-114

Wu G, Chiang JH (1996) The effect of surface compliance on foot pressure in stance. Gait Posture 4:12-129 